\title{
Distal Tibial Allograft Augmentation for Posterior Shoulder Instability Associated With Glenoid Bony Deficiency: A Case Series
}

\author{
Ron Gilat, M.D., Eric D. Haunschild, B.S., Tracy Tauro, B.S., B.A., \\ Aghogho Evuarherhe, B.S., Michael C. Fu, M.D., Anthony Romeo, M.D., \\ Nikhil Verma, M.D., and Brian J. Cole, M.D., M.B.A.
}

\begin{abstract}
Purpose: To report the clinical history and preliminary outcomes of patients who underwent posterior glenoid reconstruction using a distal tibial allograft (DTA) for the management of posterior shoulder instability with glenoid bone loss. Methods: Patients who underwent posterior shoulder stabilization with a DTA in our institution between 2011 and 2019 were retrospectively reviewed. Demographic characteristics, operative reports, and clinical and functional outcomes were recorded. Outcomes included postoperative range of motion (ROM), recurrent instability, complications, and revision surgery. All patients underwent at least 1 year of follow-up, except 2 patients who underwent revision surgery. Preoperative and postoperative ROM was compared using the 2 -tailed Student $t$ test for paired samples. Results: Ten patients who underwent DTA augmentation for posterior instability were included, comprising 2 female and 8 male patients with an average age of 24 years (range, 17-35 years). Five patients had a prior sports-related traumatic event, and 2 patients had a seizure disorder. Seven patients had undergone a prior stabilization procedure. The average reverse bony Bankart lesion was $26 \%$ of the glenoid diameter. Concomitant procedures included 4 capsular repairs, 2 labral repairs, 2 capsular plications, and 1 repair for humeral avulsion of the glenohumeral ligament. One patient reported recurrent instability after surgery. Two patients underwent revision surgery, with one removal of symptomatic hardware and one early revision owing to screw penetration into the glenoid. There was no significant difference in preoperative versus postoperative ROM. Conclusions: Posterior shoulder instability with significant bony deficiency can be managed using DTA augmentation with good outcomes and a reasonable complication rate in these challenging cases. Level of Evidence: Level IV, case series.
\end{abstract}

I solated posterior shoulder instability is less common than anterior instability, with an estimated prevalence of $2 \%$ to $10 \%$ of all shoulder dislocations. ${ }^{1-3}$

From Midwest Orthopaedics at Rush University Medical Center, Chicago, Illinois, U.S.A. (R.G., E.D.H., T.T., A.E., M.C.F., A.R., N.V., B.J.C.); and Department of Orthopaedic Surgery, Shamir Medical Center and Tel Aviv University, Tel Aviv, Israel (R.G.).

The authors report the following potential conflicts of interest or sources of funding: A.R. receives intellectual property (IP) royalties from Arthrex; receives other financial or material support from Arthrex; is a paid consultant for Arthrex; is paid presenter or speaker for Arthrex; receives research support from Arthrex and Paragen Technologies; is on the editorial or governing board of SAGE and SLACK; and receives publishing royalties and financial or material support from Saunders/Mosby-Elsevier and SLACK, outside the submitted work. N.V. is a board or committee member of American Orthopaedic Society for Sports Medicine, American Shoulder and Elbow Surgeons, and Arthroscopy Association of North America; receives research support from Arthrex, Breg, Ossur, Smith $\theta$ Nephew, and Wright Medical Technology; receives publishing royalties and financial or material support from Arthroscopy and Vindico Medical-Orthopedics Hyperguide; receives stock or stock options from Cymedica, Minivasive, and Omeros; is on the editorial or governing board of Knee and SLACK; is a paid consultant for Minivasive and Orthospace; and receives IP royalties from Smith $\theta$ Nephew; outside the
Posterior shoulder instability with posterior glenoid bone loss is rare; however, management of such cases is frequently challenging. ${ }^{4,5}$ The first line of treatment for

submitted work. B.J.C. receives IP royalties and research support from Arthrex and is a paid consultant for Arthrex. In addition, B.J.C. receives research support from Aesculap, National Institutes of Health, and Regentis; receives other financial or material support from Athletico, JRF Ortho, and Smith $\theta$ Nephew; receives IP royalties from Elsevier; receives publishing royalties and financial or material support from Operative Techniques in Sports Medicine; receives stock options from Ossio; and is a paid consultant for Regentis, outside the submitted work. Full ICMJE author disclosure forms are available for this article online, as supplementary material.

Received December 16, 2019; accepted June 8, 2020.

Address correspondence to Brian J. Cole, M.D., M.B.A., Midwest Orthopaedics at Rush University Medical Center, 1611 W Harrison St, Chicago, IL 60612,U.S.A.E-mail: brian.cole@rushortho.com

(C) 2020 THE AUTHORS. Published by Elsevier Inc. on behalf of the Arthroscopy Association of North America. This is an open access article under the CC BY-NC-ND license (http://creativecommons.org/licenses/by-nc-nd/4.0/). 2666-061X/191538

https://doi.org/10.1016/j.asmr.2020.06.005 
posterior shoulder instability is conservative treatment. ${ }^{6,7}$ However, failure of conservative treatment is not uncommon, and many patients continue to complain of vague pain, decreased athletic performance, and recurrent instability events. ${ }^{4}$ Several arthroscopic and open operative procedures have been described, including posterior capsular and labral repair, ${ }^{4,8,9}$ posterior capsular shift, rotator interval closure, subscapularis or lesser tuberosity transfer (McLaughlin and modified McLaughlin procedures), ${ }^{1,10-12}$ glenoid osteotomy, ${ }^{13,14}$ bone block, ${ }^{5,15-24}$ rotational osteotomy of the proximal humerus, ${ }^{2}$ and arthroplasty.

Posterior glenoid reconstruction using a bone block in patients with posterior glenoid deficiency has been described by several case series and case reports. ${ }^{5,15-21,23-25}$ Different bone autografts (iliac crest, acromion, and scapula) and allografts (distal tibia) have been used to reconstruct the deficient posterior glenoid. To date, literature reporting the functional outcomes of patients undergoing posterior shoulder stabilization with a distal tibial allograft (DTA) is scarce.

The purpose of this study was to report the clinical history and preliminary outcomes of patients who underwent posterior glenoid reconstruction using a DTA for the management of posterior shoulder instability with glenoid bone loss. Our hypothesis was that posterior glenoid reconstruction with a DTA would restore stability in most patients, with a low rate of recurrent instability, complications, and/or failures.

\section{Methods}

\section{Patient Population}

The study was approved by our institutional audit and review board (No. ORA19082003). An institutional database was queried to identify patients who underwent posterior glenoid reconstruction with a DTA between January 2011 and June 2019. The inclusion criteria were all patients who underwent posterior glenoid reconstruction with a DTA and had minimum l-year follow-up. The exclusion criteria were (1) patients with multidirectional instability, (2) patients who underwent posterior stabilization without a DTA, (3) patients with less than 1 year of follow-up, and (4) patients with inflammatory arthropathy.

A total of 10 patients were identified. All but 2 patients had more than 1 year of follow-up. We have therefore excluded these 2 patients with less than 1 year of follow-up from our final outcomes analysis but have reported the cases because they involved notable complications and revision surgical procedures.

\section{Data Collection}

Patient charts were reviewed, and demographic characteristics, imaging findings, surgical details, and clinical and functional outcomes were recorded. Radiographic and functional outcomes included postoperative range of motion (ROM), graft union and resorption, score on the Short Form 12 (SF-12) questionnaire, recurrent instability, complications, and revision surgery.

\section{Patient Evaluation and Indications}

All patients underwent careful preoperative assessment that included a focused history, physical examination by the operating surgeon, and imaging, including shoulder radiographs, computed tomography (CT), and magnetic resonance imaging (MRI) (Fig 1). We obtained a 3dimensional CT scan with subtraction of the humeral head in each patient, as suggested by Gupta et al. ${ }^{18}$ Indications for posterior glenoid reconstruction included recurrent instability with greater than $25 \%$ posterior glenoid bone loss and/or persistent posterior instability after failure of other stabilization procedures. Glenoid bone loss was estimated on CT scans using the best-fit circle method and verified intraoperatively using a calibrated probe and the bare area as a reference point. ${ }^{26}$ Preoperative assessment and postoperative imaging interpretation were performed unblinded as standard of care by 3 fellowship-trained orthopaedic surgeons who each had more than 20 years of experience in shoulder surgery (A.R., N.V., B.J.C.).

\section{Operative Technique}

The 3 aforementioned surgeons performed the operations in our institution. All 3 performed the surgical procedure in a similar fashion. A mini-open, arthroscopic, or combined approach was used. Patients were positioned in the lateral decubitus position, and an examination under anesthesia was performed to assess ROM and posterior, anterior, superior, and inferior instability.

Visualization of the glenohumeral joint was established using a posterior arthroscopic portal or a mini-incision posterior approach $(2-5 \mathrm{~cm})$. A formal survey of the joint was performed to assess all pathologies (Fig 2). The amount of posterior glenoid deficiency was assessed by a similar method to that described by Burkhart et al. ${ }^{27}$ The posterior glenoid was prepared through the mini-open incision or through a 7-o'clock portal, with limited dissection of the superior and medial glenoid neck to avoid injury to the suprascapular nerve.

A fresh cadaveric DTA (AlloSource, Denver, CO) was prepared in a similar fashion to that described by Gupta et al. ${ }^{18}$ In short, the lateral third of the tibial plafond was prepared to anatomically match the deficient posterior glenoid (Fig 3). Two holes were either predrilled into the graft prior to insertion to the joint or drilled over K-wires after the graft had been introduced into the joint, as it sat flush on the glenoid. The graft was introduced into the joint from a posterior mini-incision and secured to the glenoid with bicortical screws (Fig 4). Capsular and/or labral repair was performed when indicated. After copious irrigation, the wounds 

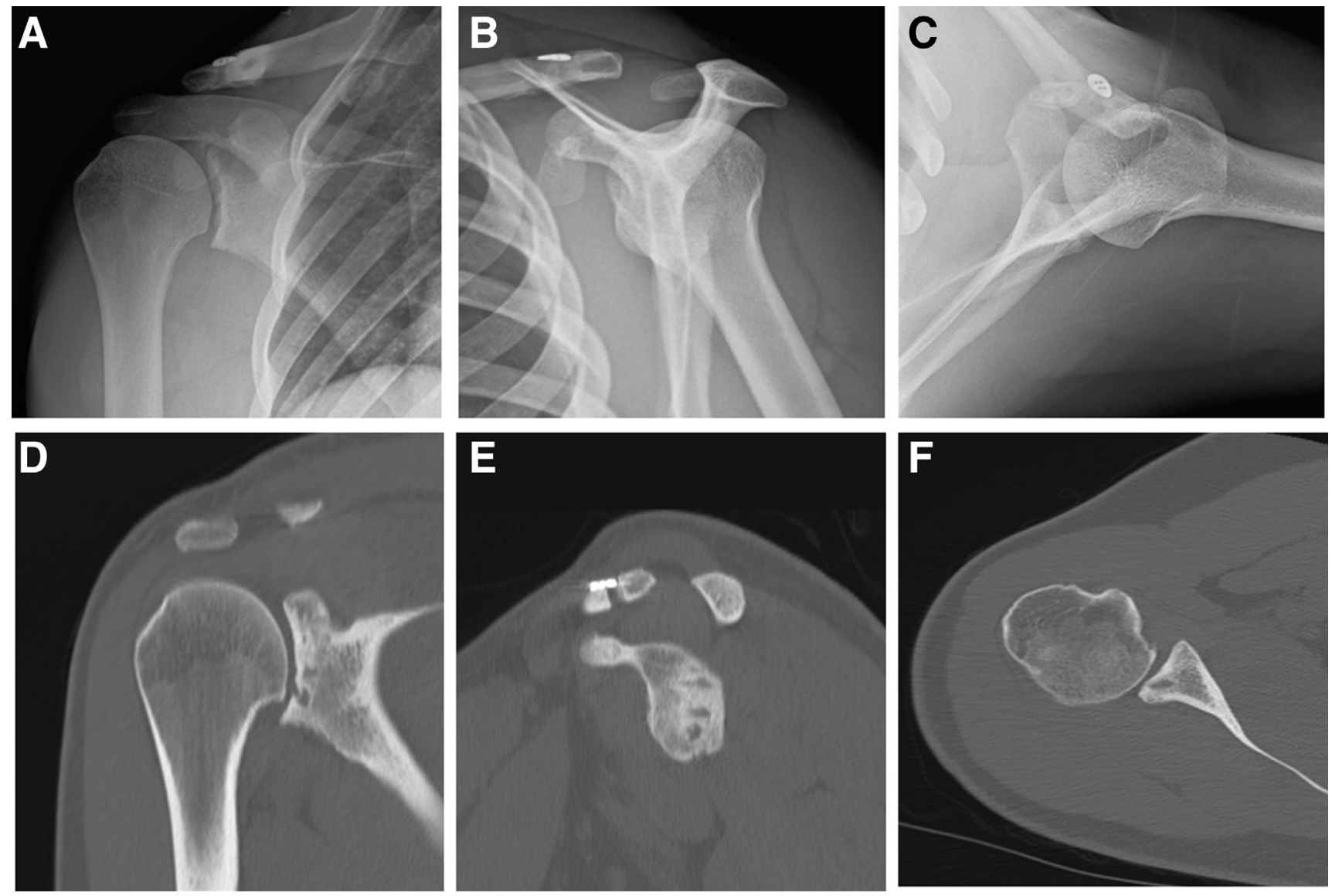

Fig 1. (A-C) Radiographs of a 25-year-old male football player, who sustained the initial injury to the right shoulder while playing football: anteroposterior (A), scapular-Y (B), and axillary (C) views. The patient initially received a diagnosis of a grade 3 acromioclavicular joint (ACJ) dislocation and was treated with ACJ reconstruction and distal clavicular excision (2010). He later received a diagnosis of posterior instability and underwent 3 soft-tissue stabilization surgical procedures (2011, 2016, and 2017). The radiographs show degenerative changes of the glenoid after distal clavicular resection, as well as a detached coracoid process, perhaps owing to failure of the ACJ reconstruction. (D-F) Computed tomography images showing degenerative changes of the glenoid and posterior subluxation of the humeral head: coronal (D), sagittal (E), and axial (F) views.

were closed in the usual fashion. Patients were placed in a gunslinger brace for approximately 6 weeks postoperatively. Postoperative radiographs were taken at first follow-up, 2 weeks postoperatively (Fig 5).

\section{Rehabilitation Protocol}

Postoperatively, the shoulder was immobilized in a shoulder brace during the day and night for the first 2 weeks and then only during the day from 2 to 6 weeks. At 3 weeks postoperatively, passive ROM was initiated, allowing up to $90^{\circ}$ of flexion, $90^{\circ}$ of abduction, and $45^{\circ}$ of internal rotation. At 6 weeks postoperatively, active and active-assisted ROM was initiated, with full active ROM expected by 12 to 16 weeks postoperatively. The timing of return to full activity was individually determined but was expected at 5 to 7 months postoperatively.

\section{Statistical Analysis}

Preoperative and postoperative ROM, including forward flexion, abduction, external rotation (with the patient's arm beside the body), and internal rotation (with the patient's hand behind the back), was compared using the 2-tailed Student $t$ test for paired samples. Internal rotation was reported according to the most superior vertebra reached with the patient's hand behind his or her back. Therefore, to compare internal rotation, vertebrae were given consecutive numbers starting at $\mathrm{Tl}$ and reaching $\mathrm{Sl}$ (e.g., $\mathrm{Tl}=1, \mathrm{Ll}=13$, and $S 1=18$ ), and statistical testing was performed accordingly. Preoperative and postoperative SF-12 scores were also compared using the 2-tailed Student $t$ test for paired samples. Statistical significance was set at $P=.05$. Stata (version 13.0; StataCorp, College Station, TX) was used for statistical analysis.

\section{Results}

A total of 10 patients underwent DTA augmentation for posterior shoulder instability with bone deficiency. This case series included 2 female and 8 male patients 

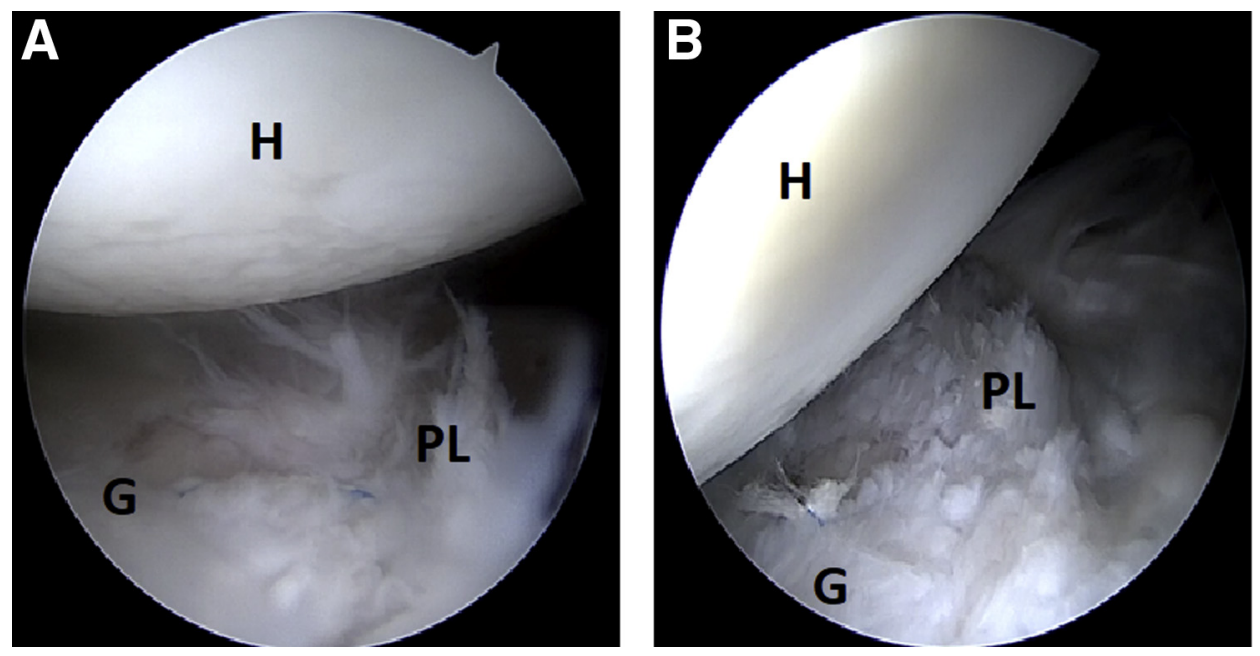

Fig 2. (A, B) Arthroscopic views from posterior portal showing posterior subluxation of humeral head $(\mathrm{H})$ and erosion and chondromalacia of posterior glenoid $(\mathrm{G})$ after repair. A prior suture anchor is visible as well. (PL, posterior labrum.)

with an average age of 24 years (range, 17-35 years). Five patients had a prior sports-related injury; of these, 3 sustained the injury during football, 1 while skiing, and 1 during volleyball. There were 2 patients with epilepsy who had recurrent posterior shoulder instability related to prior seizures. The remaining 3 patients had no definite cause of posterior instability; however, 2 of them had general hyperlaxity. An individual patient summary is presented in Table 1 , and demographic characteristics are detailed in Table 2.

Prior stabilization procedures had been performed in 7 patients $(70 \%)$; of these, 4 underwent 1 prior procedure, 1 underwent 2 prior procedures, and 2 underwent 3 prior procedures. The average reverse bony Bankart lesion was $26 \%$ of the glenoid diameter. Reverse Hill-Sachs lesions were reported during arthroscopy in 4 patients. Concomitant procedures included 5 capsular repairs, 4 labral repairs, 2 capsular plications, and 1 repair for humeral avulsion of the glenohumeral ligament (Table 3).

The mean follow-up period was $2.8 \pm 1.7$ years (range, 1.1-6.3 years). Nine patients reported restoration of the stability of the glenohumeral joint during follow-up assessments. One patient reported recurrent instability events. The preoperative ROM measures for forward flexion, abduction, external rotation, and internal rotation were $154^{\circ}, 167^{\circ}, 73^{\circ}$, and $\mathrm{T} 7$, respectively. The postoperative ROM measures were $148^{\circ}$, $149^{\circ}, 62^{\circ}$, and $\mathrm{T} 10$, respectively. There was no statistically significant difference in preoperative versus postoperative ROM, including forward flexion, abduction, external rotation, and internal rotation $(P=.63, P=.54$, $P=.24$, and $P=.23$, respectively) (Table 4 ).

Postoperative radiographs were available for 7 of the 8 patients who did not require further surgical intervention. Complete graft union was observed in 5 patients, and partial union was observed in 2. Two patients showed no graft resorption, whereas 4 patients had less than $50 \%$ resorption of the graft and 1 patient had more than $50 \%$ resorption of the graft. SF-12 scores at more than 1 year postoperatively were available for 6 patients. The mean preoperative and postoperative SF-12 physical scores (Physical Component
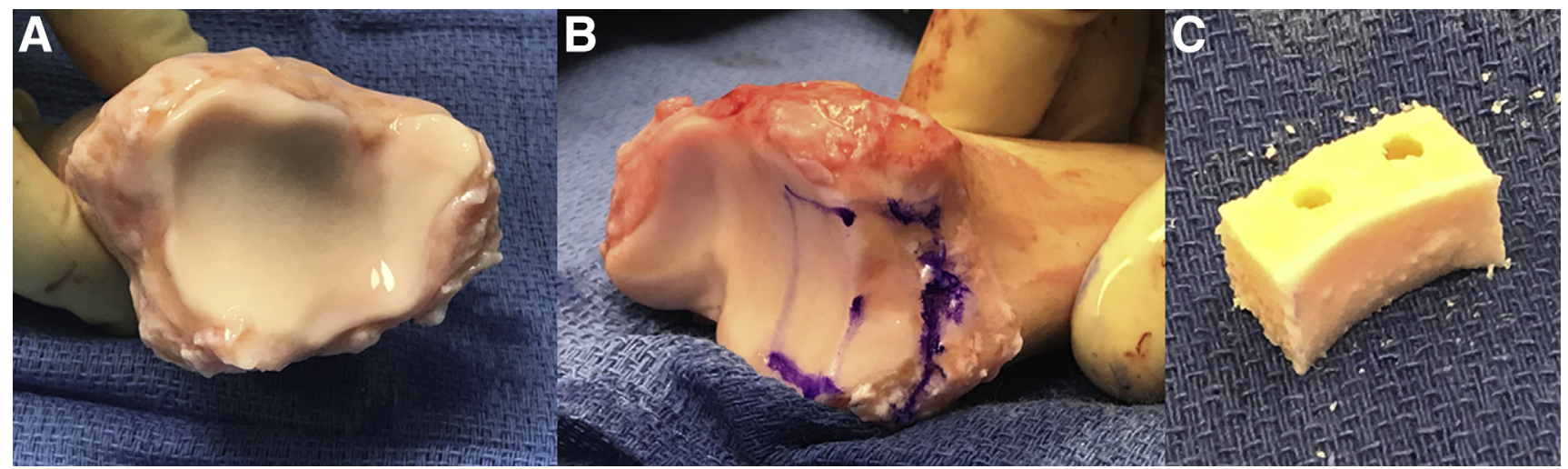

Fig 3. Distal tibial allograft preparation. (A) The articular surface is shown, which supplies the cartilaginous interface of the graft. (B) The lateral section of the articular surface is measured and marked. (C) The graft is ready for implantation; 2 holes are drilled to allow screw fixation to the glenoid with a lag screw effect. 
Fig 4. (A, B) Posterior views of completed posterior glenoid reconstruction. Suture anchors are introduced and will be used for posterior labral and capsular repair. (D, distal tibial allograft; $\mathrm{H}$, humeral head.)

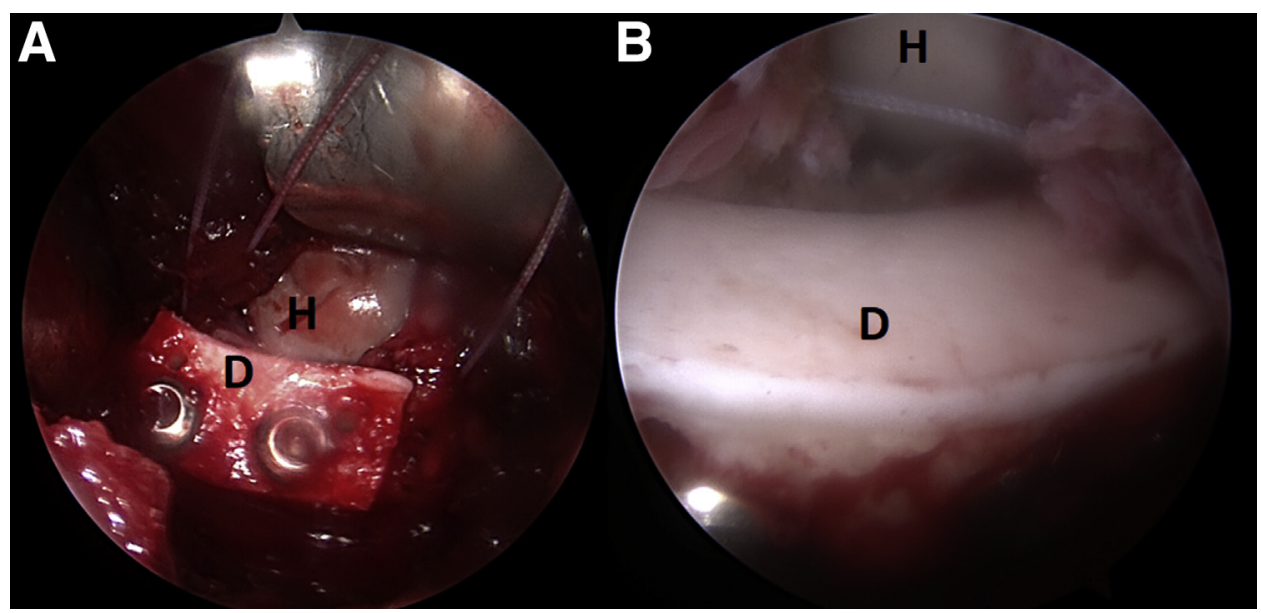

Summary) were 32.5 and 41.8 , respectively. The mean preoperative and postoperative SF-12 mental scores (Mental Component Summary) were 57.3 and 46.5, respectively. There were no statistically significant differences between preoperative and postoperative scores $(P=.25$ for physical composite scores and $P=.41$ for mental composite scores).

\section{Revision Cases}

A 24-year-old male patient injured his left shoulder while skiing in 2008. He underwent arthroscopic repair of the posterior labrum in 2012 but continued to have pain and recurrent posterior instability. CT and MRI showed evidence of posterior glenoid bone loss of approximately $30 \%$ of the glenoid diameter. Consequently, he underwent arthroscopic-assisted posterior glenoid reconstruction with a DTA, labral repair with 2 anchors, and capsulorrhaphy in 2017. On follow-up examinations, there was concern regarding screw prominence given the nature of the patient's complaints and radiographic and MRI findings. In 2018, he underwent a revision procedure with removal of the screws and debridement. He has been doing generally well since, with pain associated with certain overhead activities only.

A 21-year-old female patient with no medical history presented after posterior shoulder stabilization surgery with a posterior labral repair performed by a surgeon not affiliated with our institution. The patient had hyperlaxity of the right shoulder and was able to voluntarily dislocate the right shoulder posteriorly only. She underwent a revision arthroscopic shoulder stabilization procedure with a labral repair using 3 anchors. She subsequently returned to the clinic with persistent posterior instability and underwent posterior shoulder stabilization with a DTA by a mini-open approach. She returned 2 weeks later for standard postoperative radiographs, which raised the suspicion of screw penetration into the glenoid articular surface. CT confirmed the diagnosis and showed glenoid retroversion and
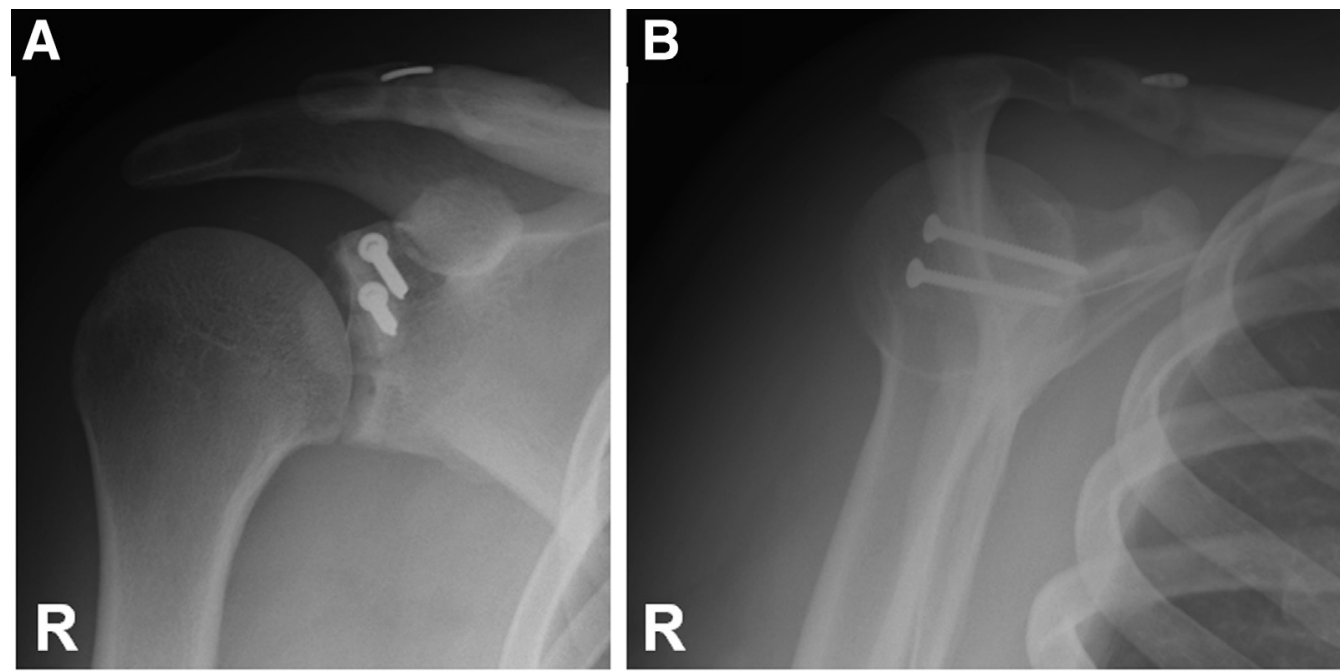

Fig 5. Postoperative followup radiographs 2 weeks after posterior glenoid reconstruction: anteroposterior (A) and scapular (B) views. ( $\mathrm{R}$, right.) 
Table 1. Summary of Patients

\begin{tabular}{|c|c|c|c|c|c|c|c|c|c|c|c|c|}
\hline Patient No. & $\begin{array}{c}\text { Age at } \\
\text { Surgery, } \\
\text { yr }\end{array}$ & Sex & $\begin{array}{l}\text { Dominant } \\
\text { Arm }\end{array}$ & Hyperlaxity & $\begin{array}{l}\text { Seizure } \\
\text { Disorder }\end{array}$ & Comorbidities & Sport & $\begin{array}{c}\text { No. } \\
\text { of Prior } \\
\text { Surgical } \\
\text { Procedures }\end{array}$ & $\begin{array}{l}\text { Concomitant } \\
\text { Procedures }\end{array}$ & $\begin{array}{l}\text { Recurrent } \\
\text { Instability }\end{array}$ & Complications & $\begin{array}{c}\text { Time } \\
\text { to Last } \\
\text { Follow-Up, } \\
\text { yr }\end{array}$ \\
\hline \multicolumn{13}{|l|}{$\begin{array}{l}\text { Non-revision } \\
\text { cases }\end{array}$} \\
\hline 1 & 17 & Female & Yes & Yes & No & - & - & 3 & $\begin{array}{l}\text { Posterior capsular repair } \\
\text { for reverse HAGL }\end{array}$ & Yes & - & 4.6 \\
\hline 2 & 25 & Male & Yes & No & No & $\begin{array}{l}\text { Thoracic outlet syndrome } \\
\text { treated surgically }\end{array}$ & Football & 3 & Posterior capsular repair & No & - & 1.3 \\
\hline 3 & 19 & Male & Yes & No & No & - & Football & 1 & Posterior capsular repair & No & - & 2.1 \\
\hline 4 & 35 & Male & Yes & No & No & - & - & 0 & Posterior labral repair & No & - & 1.7 \\
\hline 5 & 19 & Male & Yes & No & No & - & Volleyball & 1 & $\begin{array}{l}\text { Posterior capsular } \\
\text { plication }\end{array}$ & No & - & 6.3 \\
\hline 6 & 27 & Male & Yes & Yes & Yes & Epilepsy & - & 0 & Posterior labral repair & No & - & 3.8 \\
\hline 7 & 33 & Male & Yes & No & Yes & $\begin{array}{l}\text { Epilepsy, cerebral } \\
\text { aneurysms }\end{array}$ & - & 0 & $\begin{array}{l}\text { Posterior capsular and } \\
\text { labral repair }\end{array}$ & No & - & 2.2 \\
\hline 8 & 21 & Male & No & No & No & - & Football & 1 & $\begin{array}{l}\text { Posterior capsular } \\
\text { plication }\end{array}$ & No & - & 1.1 \\
\hline \multicolumn{13}{|l|}{ Revision cases } \\
\hline 9 & 21 & Female & Yes & Yes & No & - & - & 2 & Posterior capsular repair & No & $\begin{array}{l}\text { Screw penetration } \\
\text { into glenoid }\end{array}$ & $\begin{array}{c}\text { Early revision } \\
\text { (at } 3 \text { weeks } \\
\text { postop) }\end{array}$ \\
\hline 10 & 24 & Male & No & No & No & $\begin{array}{l}\text { Asthma, Arnold-Chiari } \\
\text { malformation }\end{array}$ & Skiing & 1 & Posterior labral repair & No & Prominent screws & $\begin{array}{c}\text { Early revision } \\
\text { (at } 4 \text { mo } \\
\text { postop) }\end{array}$ \\
\hline
\end{tabular}

HAGL, humeral avulsion of glenohumeral ligament; postop, postoperatively. 
Table 2. Demographic Characteristics

\begin{tabular}{lc}
\hline & Data \\
\hline $\mathrm{N}$ & 10 \\
Age, yr & 24 \\
$\quad$ Mean & $17-35$ \\
$\quad$ Range & \\
Sex, n (\%) & $2(20)$ \\
$\quad$ Female & $8(80)$ \\
Male & \\
Side, n (\%) & $8(80)$ \\
Right & $2(20)$ \\
Left & $8(80)$ \\
Dominant hand affected, n (\%) & \\
BMI & 28.0 \\
Mean & $21.7-39.2$ \\
$\quad$ Range & $3(30)$ \\
Hyperlaxity, n (\%) & $2(20)$ \\
Seizure disorder, n (\%) & $5(50)$ \\
Sports-related traumatic event, n (\%) & $7(70)$ \\
Prior stabilization surgery, n (\%) & \\
No. of prior surgical procedures, n & 3 \\
0 & \\
1 & 4 \\
2 & 1 \\
3 & 2 \\
\hline BMI body mass index, &
\end{tabular}

possible hypoplasia. Soon after, the patient underwent revision DTA augmentation with screw repositioning and a new DTA. Further follow-up of this patient is unavailable because the last procedure was only recently performed.

\section{Discussion}

The main finding of this study was that for isolated posterior shoulder instability accompanied by glenoid bone deficiency, posterior glenoid augmentation with a DTA is safe and effective in restoring stability in these challenging cases. Moreover, we found that ROM was not compromised after the procedure. However, 2 patients did require revision surgery because of hardware failure, and an additional patient reported a sensation of recurrent instability. In addition, although the SF-12 physical scores did improve postoperatively (from 32.5 to 41.8 ), this finding was not statistically significant.

Bone-block reconstruction can be performed using an autograft or allograft. Available autografts that have been described for the treatment of posterior shoulder instability with bone loss include iliac crest, acromial, and scapular autografts. ${ }^{15-17,19,20,25}$

The iliac crest is the most commonly reported donor site. Barbier et al. ${ }^{19}$ reported a case series of 8 patients who underwent posterior stabilization with an autologous iliac crest bone block. All procedures were performed by 1 senior author using an open posterior L-shaped approach. The authors reported that stability was established in all patients and the mean postoperative Constant score (96.3) was greater than the mean preoperative score (82.5). However, patients were hospitalized for a mean of 6 days, 3 patients underwent a later operation for screw removal, and 1 patient experienced hematoma at the donor site.

Mowery et al. ${ }^{28}$ studied 5 patients who underwent stabilization using an iliac crest autograft by an open approach. A subsequent anterior dislocation occurred in 1 patient, and 2 patients underwent "scar revision" surgery. Servien et al. ${ }^{15}$ used an open approach to perform glenoid reconstruction with iliac crest autograft in 20 patients (21 shoulders) with posterior shoulder instability. They reported that 15 patients returned to their preinjury level. One patient had recurrent posterior dislocation, and 2 patients exhibited substantial posterior apprehension. Levigne et al. ${ }^{29}$ published a series of 29 patients ( 31 shoulders) after open posterior iliac crest bone-block procedures. They reported that $39 \%$ of patients had persistent pain; there was a $13 \%$ recurrent instability rate and $23 \%$ graft lysis rate.

Compared with the aforementioned studies, our study shows a similar rate of restoration of shoulder stability and a similar reoperation rate. Although only 2 patients in this study required revision surgery owing to hardware failure and an additional patient complained of recurrent instability, this is a high revision rate in a small group of subjects with limited follow-up. It is important to note that this study differs from other studies in that a mini-open and/or arthroscopic approach was performed, patients were discharged on the same day as surgery, and there was no donor-site morbidity associated with the use of DTA.

Not all studies published on the use of iliac crest bone block have shown satisfactory results. Meuffels et al. ${ }^{20}$ reported a long-term outcome study (median, 18 years) on 11 patients who underwent stabilization with an iliac crest bone block using an open posterior

Table 3. Surgical Details

\begin{tabular}{lc}
\hline & Data \\
\hline Reverse Hill-Sachs lesion, n & 4 \\
Reverse bony Bankart lesion, n & 10 \\
Average size of reverse Bankart lesion, \%* & 26 \\
Concomitant pathology, n & \\
$\quad$ Posterior labral tear & 2 \\
$\quad$ HAGL & 1 \\
Concomitant procedure, n & \\
$\quad$ Posterior labral repair & 4 \\
HAGL repair & 1 \\
$\quad$ Capsular repair & 5 \\
Capsular plication & 2 \\
Average graft width (range), mm & $10.2(7-15)$ \\
Average graft length (range), mm & $(15-25)$ \\
No. of screws used for fixation, n & 1 \\
$\quad$ l & \\
2 & 9 \\
\hline HAGL, humeral avulsion of glenohumeral ligament. \\
$\quad$ *Percentage of posterior glenoid loss relative to maximal glenoid \\
horizontal diameter.
\end{tabular}


Table 4. Preoperative and Postoperative Range of Motion

\begin{tabular}{lcc}
\hline & Preoperative & Postoperative \\
\hline Forward flexion, $^{\circ}$ & $154(90-180)$ & $148(90-180)$ \\
Abduction, $^{\circ}$ & $167(120-180)$ & $149(70-180)$ \\
External rotation, $^{\circ}$ & $73(45-100)$ & $62(40-90)$ \\
Internal rotation $^{\circ}$ & Approximately T7 & Approximately T10 \\
& $(\mathrm{T} 2-\mathrm{S} 1)$ & $($ T4-L4) \\
\hline
\end{tabular}

NOTE. Data are presented as mean (range).

approach. Of the patients, 5 (45\%) said that they would not elect to undergo the operation again; moreover, instability remained in 4 patients $(36 \%)$. The clinical outcome scores had also deteriorated at a median of 18 years' follow-up. However, the study by Meuffels et al. presented a series in which many patients were treated more than 2 decades ago. Surgical techniques likely have developed and improved since then, and therefore, the outcomes of procedures performed today may differ.

Wellman et al. ${ }^{16}$ reported on 18 patients (24 shoulders) who underwent arthroscopic posterior iliac crest bone-block stabilization. They found recurrent instability in 3 patients $(12.5 \%)$, and 7 patients underwent a revision procedure. They reported 1 complication in 1 patient requiring graft repositioning, as well as 1 patient with postoperative pneumothorax. Of the patients, 16 $(67 \%)$ were required to undergo hardware removal of screws.

Other options include the use of scapular spine or pedicled acromial autograft. ${ }^{17,25,30,31}$ The French Society of Arthroscopy has been supporting 2 studies using an arthroscopic acromial pedicled bone-block technique. ${ }^{31,32}$ Presumably, this technique provides a softtissue sling effect, similar to that seen with the Latarjet procedure; this occurs as a result of the attachment of the deltoid to the graft. The studies' results suggest a lower occurrence of osteoarthritis with the pedicled acromial graft than with iliac bone graft, but evidence regarding long-term outcomes is limited. ${ }^{31,32}$

Donor-site morbidity could be a serious burden after an operation involving an autograft. Iliac crest harvesting is associated with gait disturbance within the first few weeks, significant pain, risk of injury to the lateral femoral cutaneous nerve, risk of serious infection including osteomyelitis, and an additional scar. ${ }^{33}$ On the other hand, the use of allografts can be associated with higher costs and limited availability in some geographic regions.

Two technique articles have described the use of posterior glenoid reconstruction with a DTA., ${ }^{5,18}$ All 3 patients described in these technique articles were reported to have good and stable shoulder function and improvement in pain, with more than 1 year of followup. Both technique articles and our case series describe the use of screws for graft fixation. Of note, Boileau et al. ${ }^{24}$ have described a technique for posterior boneblock fixation using suture anchor fixation. This technique may decrease the incidence of symptomatic hardware requiring screw removal, as occurred in 1 patient in our series.

Nacca et al. ${ }^{34}$ performed a cadaveric study comparing the peak force required to translate the humerus beyond the glenoid lip and lateral displacement during posterior translation between a DTA and a scapular spinal autograft for posterior shoulder instability with glenoid bone loss. They found that both grafts effectively restored stability, with no differences in peak force or lateral displacement. Frank et al. ${ }^{35}$ compared glenohumeral contact pressures and contact areas after posterior glenoid reconstruction with an iliac crest bone graft or a DTA in 8 cadaveric shoulders. No differences in contact pressures or peak forces were found when they compared both the iliac crest bone graft and the DTA with the intact glenoid $(P>.05$ in all cases). They concluded that a DTA confers similar contact mechanics to an iliac crest bone graft, with the possible advantages of an anatomic reconstruction, resulting in a cartilaginous, congruent articulation with the humeral head.

\section{Limitations}

The main limitations of our study are the small sample size, limited follow-up period, and lack of jointspecific patient-reported outcomes. With that in mind, the literature regarding the use of DTA for the management of this rare pathology is scarce and, therefore, we believe these preliminary findings are valuable. Only 8 of the 10 patients presented in this study had postoperative radiographs available, which may affect reported rates of graft resorption, union, and hardware failure. The findings of the statistical analyses performed to compare preoperative versus postoperative ROM and SF-12 scores should be regarded only as suggestive because this small sample of patients does not constitute enough power to support the hypothesis that there is no difference between the 2 paired variables. Moreover, we did not report clinically significant outcomes $^{36}$ because these had yet to be established for posterior shoulder instability and particularly for this rare procedure. Finally, we did not report joint-specific patient-reported outcomes because of the need for early revision in 2 patients and the low compliance and unavailability of several other patients. However, we did report the SF-12 scores with at least 1 year of follow-up for 6 patients.

\section{Conclusions}

Posterior shoulder instability with significant bony deficiency can be managed using DTA augmentation with good outcomes and a reasonable complication rate in these challenging cases. 


\section{References}

1. McLaughlin HL. Posterior dislocation of the shoulder. J Bone Joint Surg Am 1952;34:584-590.

2. Boyd HB, Sisk TD. Recurrent posterior dislocation of the shoulder. J Bone Joint Surg Am 1972;54:779-786.

3. Antoniou J, Duckworth DT, Harryman DT. Capsulolabral augmentation for the management of posteroinferior instability of the shoulder. J Bone Joint Surg Am 2000;82: 1220.

4. Provencher MT, LeClere LE, King S, et al. Posterior instability of the shoulder: Diagnosis and management. Am J Sports Med 2011 1;39:874-886.

5. Millett PJ, Schoenahl J-Y, Register B, Gaskill TR, van Deurzen DF, Martetschläger F. Reconstruction of posterior glenoid deficiency using distal tibial osteoarticular allograft. Knee Surg Sports Traumatol Arthrosc 2013;21: 445-449.

6. Fronek J, Warren R, Bowen M. Posterior subluxation of the glenohumeral joint. J Bone Joint Surg Am 1989;71: 205-216.

7. Schwartz E, Warren R, O'Brien S, Fronek J. Posterior shoulder instability. Orthop Clin North Am 1987;18:409.

8. Bradley JP, McClincy MP, Arner JW, Tejwani SG. Arthroscopic capsulolabral reconstruction for posterior instability of the shoulder: A prospective study of 200 shoulders. Am J Sports Med 2013;41:2005-2014.

9. Savoie FH III, Holt MS, Field LD, Ramsey JR. Arthroscopic management of posterior instability: Evolution of technique and results. Arthroscopy 2008;24:389-396.

10. Martetschläger F, Padalecki JR, Millett PJ. Modified arthroscopic McLaughlin procedure for treatment of posterior instability of the shoulder with an associated reverse Hill-Sachs lesion. Knee Surg Sports Traumatol Arthrosc 2013;21:1642-1646.

11. Krackhardt T, Schewe B, Albrecht D, Weise K. Arthroscopic fixation of the subscapularis tendon in the reverse Hill-Sachs lesion for traumatic unidirectional posterior dislocation of the shoulder. Arthroscopy 2006;22:227. el-227.e6.

12. Hawkins R, Pianta R, Mendoza F. Locked posterior dislocation of the shoulder. J Bone Joint Surg Am 1987;69:9-18.

13. Hawkins RH. Glenoid osteotomy for recurrent posterior subluxation of the shoulder: Assessment by computed axial tomography. J Shoulder Elbow Surg 1996;5:393-400.

14. Lacheta L, Singh TS, Hovsepian JM, Braun S, Imhoff AB, Pogorzelski J. Posterior open wedge glenoid osteotomy provides reliable results in young patients with increased glenoid retroversion and posterior shoulder instability. Knee Surg Sports Traumatol Arthrosc 2019;27:299-304.

15. Servien E, Walch G, Cortes ZE, Edwards TB, O'Connor DP. Posterior bone block procedure for posterior shoulder instability. Knee Surg Sports Traumatol Arthrosc 2007;15:1130-1136.

16. Wellmann M, Pastor M-F, Ettinger M, Koester K, Smith T. Arthroscopic posterior bone block stabilization-early results of an effective procedure for the recurrent posterior instability. Knee Surg Sports Traumatol Arthrosc 2018;26: 292-298.

17. Métais P, Grimberg J, Clavert P, et al. Posterior shoulder instability managed by arthroscopic acromial pediculated bone-block. Technique. Orthop Traumatol Surg Res 2017;103:S203-S206.

18. Gupta AK, Chalmers PN, Klosterman E, Harris JD, Provencher MT, Romeo AA. Arthroscopic distal tibial allograft augmentation for posterior shoulder instability with glenoid bone loss. Arthrosc Tech 2013;2:e405-e411.

19. Barbier O, Ollat D, Marchaland J-P, Versier G. Iliac boneblock autograft for posterior shoulder instability. Orthop Traumatol Surg Res 2009;95:100-107.

20. Meuffels D, Schuit H, Van Biezen F, Reijman M, Verhaar J. The posterior bone block procedure in posterior shoulder instability: A long-term follow-up study. J Bone Joint Surg Br 2010;92:651-655.

21. Lafosse L, Franceschi G, Kordasiewicz B, Andrews WJ, Schwartz D. Arthroscopic posterior bone block: Surgical technique. Musculoskelet Surg 2012;96:205-212.

22. Cerciello S, Visonà E, Morris BJ, Corona K. Bone block procedures in posterior shoulder instability. Knee Surg Sports Traumatol Arthrosc 2016;24:604-611.

23. Smith T, Goede F, Struck M, Wellmann M. Arthroscopic posterior shoulder stabilization with an iliac bone graft and capsular repair: A novel technique. Arthrosc Tech 2012;1:e181-e185.

24. Boileau P, Hardy M-B, McClelland WB Jr, Thélu C-E, Schwartz DG. Arthroscopic posterior bone block procedure: A new technique using suture anchor fixation. Arthrosc Tech 2013;2:e473-e477.

25. Struck M, Wellmann M, Becher C, Pastor M, Smith T. Results of an open posterior bone block procedure for recurrent posterior shoulder instability after a short-and long-time follow-up. Knee Surg Sports Traumatol Arthrose 2016;24:618-624.

26. Piasecki DP, Verma NN, Romeo AA, Levine WN, Bach BR Jr, Provencher MT. Glenoid bone deficiency in recurrent anterior shoulder instability: Diagnosis and management. J Am Acad Orthop Surg 2009; 17:482-493.

27. Burkhart SS, DeBeer JF, Tehrany AM, Parten PM. Quantifying glenoid bone loss arthroscopically in shoulder instability. Arthroscopy 2002;18:488-491.

28. Mowery C, Garfin S, Booth R, Rothman R. Recurrent posterior dislocation of the shoulder: Treatment using a bone block. J Bone Joint Surg Am 1985;67:777-781.

29. Levigne C, Garret J, Walch G. Posterior bone block for posterior instability. Tech Shoulder Elbow Surg 2005;6:26-35.

30. Kouvalchouk J, Coudert X, Watin LA, Da Silva Rosa R, Paszkowski A. Treatment of posterior instability of the shoulder joint using an acromial stop with a pediculated deltoid flap. Rev Chir Orthop Reparatrice Appar Mot 1993;79: 661-665 [in French].

31. Sirveaux F, Leroux J, Roche O, Gosselin O, De MG, Molé D. Surgical treatment of posterior instability of the shoulder joint using an iliac bone block or an acromial pediculated bone block: Outcome in eighteen patients. Rev Chir Orthop Reparatrice Appar Mot 2004;90:41 1-419 [in French].

32. DiMaria S, Bokshan SL, Nacca C, Owens B. History of surgical stabilization for posterior shoulder instability. JSES Open Access 2019;3:350-356.

33. Costa LM, Sauvigne T, Guiol J. Morbidity of autologous bone harvesting in implantology: Literature review from 1990 to 2015. Rev Stomatol Chir Maxillofac Chir Orale 2016;117:388-402 [in French]. 
34. Nacca C, Gil JA, DeFroda SF, Badida R, Owens BD. Comparison of a distal tibial allograft and scapular spinal autograft for posterior shoulder instability with glenoid bone loss. Orthop J Sports Med 2018;6: 2325967118786697.

35. Frank RM, Shin J, Saccomanno MF, et al. Comparison of glenohumeral contact pressures and contact areas after posterior glenoid reconstruction with an iliac crest bone graft or distal tibial osteochondral allograft. Am J Sports Med 2014;42:2574-2582.

36. Harris JD, Brand JC, Cote MP, Faucett SC, Dhawan A. Research pearls: The significance of statistics and perils of pooling. Part 1: Clinical versus statistical significance. Arthroscopy 2017;33:1102-1112. 